Bull. Austral. Math. Soc.

30c80, 30D40, 30н05

Vol. 44 (1991) [527-528]

\title{
A REMARK ON BOUNDEDNESS OF BLOCH FUNCTIONS
}

\section{KRZYSZTOF SAMOTIJ}

Two consequences of a theorem of Dahlberg are derived. Let $f$ be a holomorphic function in the unit disk $D$ of the complex plane, and let $E$ be an $F_{\sigma}$ subset of the unit circle T. Suppose that $\varlimsup_{r \rightarrow 1-}|f(r w)| \leqslant M, w \in T \backslash E$, for some constant $M$. Then $f$ is bounded in either of the two cases:

(i) if $f$ is in the Bloch space and $E$ is of zero measure with respect to the Hausdorff measure associated with the function $\psi(t)=t \log \log \left(2 \pi e^{e} / t\right)$,

(ii) if $f$ is integrable with respect to the planar Lebesgue measure on $\mathbf{D}$ and $E$ is of zero measure with respect to the Hausdorff measure associated with the function $\psi(t)=t \log \left(2 \pi e^{e} / t\right)$.

Let $\mathbf{D}$ be the unit disk of the complex plane, and let $\mathbf{T}$ be the unit circle. For a function $\psi$ satisfying the usual conditions, let $\Lambda_{\psi}$ be the Hausdorff measure on $\mathbf{T}$ corresponding to $\psi$. Let $\phi_{1}(t)=\log \left(2 \pi e^{e} / t\right), \phi_{2}(t)=\log \left[\phi_{1}(t)\right], 0<t \leqslant 2 \pi$, and let $\psi_{j}(t)=t \phi_{j}(t), j=1,2$.

The following theorem is a modification of a theorem of Dahlberg [2, Theorem 4], and can be proved in a very similar manner.

Theorem. (Dahlberg). Let $j=1$ or $j=2$. Let $E \subset \mathrm{T}$ be an $F_{\sigma}$ set with $\Lambda_{\psi_{j}}(E)=0$. Let $u$ be a sub-harmonic function on D. Suppose that there are constants $C$ and $M$ such that

$$
u(z) \leqslant C \phi_{j}(1-|z|)
$$

for all $z \in \mathbf{D}$, and

$$
\varlimsup_{r \rightarrow 1-} u(r w) \leqslant M
$$

if $w \in \mathbf{T} \backslash E$. Then the function $u$ is bounded above in $\mathbf{D}$.

Let $B$ denote the Bloch space, that is, the space of those holomorphic functions $f$ on $\mathbf{D}$ which satisfy the condition

$$
\sup _{z \in D}\left(1-|z|^{2}\right)\left|f^{\prime}(z)\right|<+\infty \text {. }
$$

Since for any $f \in B$ the function $u=\log |f|$ is sub-harmonic on $\mathbf{D}$ and satisfies (A) with $j=2$ and some constant $C$, we have the following corollary, which is a generalisation of $[1$, Theorem 2].

Received 31 January 1991

Copyright Clearance Centre, Inc. Serial-fee code: 0004-9729/91 \$A2.00+0.00. 
Corollary 1. Let $f \in B$. Suppose that there are: a constant $M$ and an $F_{\sigma}$ set $E \subset \mathbf{T}$ with $\Lambda_{\psi_{2}}(E)=0$ such that

$$
\varlimsup_{r \rightarrow 1-}|f(r w)| \leqslant M
$$

for $w \in \mathbf{T} \backslash E$. Then $f$ is bounded in $\mathbf{D}$.

Now, let us observe that if $f$ is a holomorphic function in $D$ and $\iint_{x^{2}+y^{2}<1}|f(x+i y)| d x d y<+\infty$, then by the mean value property we have

$$
\begin{aligned}
|f(z)| & =\left|\frac{1}{\pi(1-|z|)^{2}} \iint_{|x+i y-z|<1-|z|} f(x+i y) d x d y\right| \\
& \leqslant \frac{1}{\pi(1-|z|)^{2}} \iint_{x^{2}+y^{2}<1}|f(x+i y)| d x d y .
\end{aligned}
$$

Therefore the subharmonic function $u=\log |f|$ satisfies (A) with $j=1$ and some constant $C$. Thus we have

COROLlary 2. Let $f$ be a holomorphic function in $\mathbf{D}$ integrable with respect to the planar Lebesgue measure on $\mathrm{D}$. Suppose that there are: a constant $M$ and an $F_{\sigma}$ set $E \subset \mathbf{T}$ with $\Lambda_{\psi_{1}}(E)=0$ such that

$$
\varlimsup_{r \rightarrow 1-}|f(r w)| \leqslant M
$$

for $w \in \mathbf{T} \backslash E$. Then $f$ is bounded in $\mathbf{D}$.

Taking $E=\emptyset$ in Corollary 2 we obtain the affirmative answer to a question asked in $[1, \mathrm{p} .37]$. For a finite set $E$, Corollary 2 proves a conjecture from [3].

\section{REFERENCES}

[1] A. Bonilla and F. Perez Gonzales, 'Radial growth and boundedness for Block functions', Bull. Austral. Math. Soc. 42 (1990), 33-39.

[2] B.E.I. Dahlberg, 'On the radial boundary values of subharmonic functions', Math. Scand. 40 (1977), 301-317.

[3] R.C. Goolsby, 'Boundedness for Bloch functions', Rocky Mountain J. Math. 16 (1986), 717-726. 